\title{
Gauging the full $\boldsymbol{R}$-symmetry group in five-dimensional, $\mathcal{N}=2$ Yang-Mills-Einstein-tensor supergravity
}

\author{
M. Günaydin* \\ CERN, Theory Division, 1211 Geneva 23, Switzerland \\ and Physics Department, Penn State University, University Park, Pennsylvania 16802 \\ M. Zagermann ${ }^{\dagger}$ \\ Physics Department, Penn State University, University Park, Pennsylvania 16802
}

(Received 4 May 2000; published 20 February 2001)

\begin{abstract}
We show that certain five-dimensional, $\mathcal{N}=2$ Yang-Mills-Einstein supergravity theories admit the gauging of the full $R$-symmetry group, $S U(2)_{R}$, of the underlying $\mathcal{N}=2$ Poincare superalgebra. This generalizes the previously studied Abelian gaugings of $U(1)_{R} \subset S U(2)_{R}$, and completes the construction of the most general vector and tensor field coupled five-dimensional, $\mathcal{N}=2$ supergravity theories with gauge interactions. The gauging of $S U(2)_{R}$ turns out to be possible only in special cases, and leads to a new type of scalar potential. For a large class of these theories the potential does not have any critical points.
\end{abstract}

DOI: 10.1103/PhysRevD.63.064023

\section{INTRODUCTION}

Five-dimensional gauged supergravity theories have been subject to a renewed intense interest during the last three years. They offer an important tool in the study of the AdS conformal field theory (CFT) correspondence [1-4] and have, more recently, been discussed as a potential framework for an embedding of the Randall-Sundrum (RS) scenario $[5,6]$ into string or M theory.

Whereas the embedding of the original discontinuous RSmodel [5] into $5 \mathrm{D}, \mathcal{N}=2$ gauged pure supergravity on $\mathrm{R}^{4} \times S^{1} / Z_{2}$ was studied in [7-9], a realization in terms of a smooth ('thick') Bogomol'nyi-Prasad-Sommerfield (BPS) domain wall solution seemed to be incompatible with a variety of scalar potentials of known matter coupled $\mathcal{N}=2$ supergravity theories [10-12].

Since the most general $5 \mathrm{D}, \mathcal{N}=2$ gauged supergravity theory has not yet been constructed, ${ }^{1}$ it is, however, still unclear how general these no-go theorems really are. A construction of the most general types of these theories should therefore help to settle this question, and might also be interesting for (bulk-)matter coupled generalizations of the discontinuous model of [5,7-9]. At the same time, a complete knowledge of $\mathcal{N}=2$ gauged supergravity theories might also contribute to a better understanding of various aspects of the $\mathcal{N}=8$ theory (such as, e.g., the structure of its vacua) with possible implications for the AdS-CFT correspondence.

Motivated by these and other applications, we have recently studied the possible gaugings of vector and tensor field coupled $5 \mathrm{D}, \mathcal{N}=2$ supergravity theories. All these theories (including the ones involving tensor multiplets) can be derived from the ungauged $\mathcal{N}=2$ Maxwell-Einstein supergravity theories (MESGT's) of Ref. [13]. These theories de-

\footnotetext{
*Email address: murat@ phys.psu.edu

${ }^{\dagger}$ Email address: zagerman@ phys.psu.edu

${ }^{1}$ See note added.
}

PACS number(s): 04.65.+e, 04.50.+h, 11.15. - q scribe the coupling of Abelian vector multiplets to $\mathcal{N}=2$ supergravity and have a global symmetry group of the form $S U(2)_{R} \times G$. Here, $G$ is the subgroup of the isometry group of the scalar field target space that extends to a symmetry group of the full Lagrangian, and $S U(2)_{R}$ denotes the automorphism group (" $R$-symmetry group") of the $5 \mathrm{D}, \mathcal{N}=2$ Poincaré superalgebra.

In [14] we generalized the earlier work [15] and constructed all possible gaugings of subgroups of $U(1)_{R} \times G$, where $U(1)_{R} \subset S U(2)_{R}$ denotes the Abelian subgroup of $S U(2)_{R}$. In particular, we also covered the case when the gauging of a subgroup of $G$ involves the dualization of some of the vector fields of the ungauged theory to "self-dual" [16] antisymmetric tensor fields, a mechanism that is well known from the maximally extended gauged supergravities in $d=7$ [17] and $d=5[18-20]$ dimensions.

Thus, the only gaugings that have not yet been covered in this framework are those involving gaugings of the full $R$-symmetry group $S U(2)_{R}$. It is the purpose of this paper to close this gap. This will complete the construction of the possible gaugings of the entire vector-tensor sector of $\mathcal{N}$ $=2$ matter coupled supergravity theories in five dimensions.

The outline of this paper is as follows. In Sec. II, we first analyze to what extent the full $R$-symmetry group $S U(2)_{R}$ can be gauged within the framework of vector and tensor field coupled $5 \mathrm{D}, \mathcal{N}=2$ supergravity theories. The corresponding Lagrangians and the supersymmetry transformation rules are then derived via the Noether method starting from some of our earlier results [14]. Section III, finally, is devoted to a discussion of the resulting scalar potentials.

\section{GAUGING THE FULL $R$-SYMMETRY GROUP $S U(2)_{R}$}

The gauging of $S U(2)_{R}$ is a little less straightforward than gaugings of subgroups of $U(1)_{R} \times G$, as we shall now explain.

The supermultiplets we are dealing with are $(\mu, \nu, \ldots$ 
and $m, n, \ldots$ denote curved and flat spacetime indices, respectively):

(i) The $\mathcal{N}=2$ supergravity multiplet, containing the graviton (fünfbein) $e_{\mu}^{m}$, two gravitini $\Psi_{\mu}^{i}(i, j, \ldots,=1,2)$ and one vector field $A_{\mu}$

(ii) The $\mathcal{N}=2$ vector multiplet, comprising one vector field $A_{\mu}$, two spin-1/2 fermions $\lambda^{i}(i, j, \ldots 1,2)$ and one real scalar field $\varphi$

(iii) The $\mathcal{N}=2$ "self-dual" tensor multiplet consisting of two real two-form fields $B_{\mu \nu}^{(1)}, B_{\mu \nu}^{(2)}$; four spin-1/2 fermions $\lambda^{(1) i}, \lambda^{(2) i}(i, j, \ldots=1,2)$ and two real scalar fields $\varphi^{(1)}$, $\varphi^{(2)}$.

Of all the above fields, only the gravitini and the spin-1/2 fermions transform non-trivially under $S U(2)_{R}$ (they form doublets labeled by the index $i=1,2)$. In particular, all the vector fields are singlets under $S U(2)_{R}$. In order to gauge a non-Abelian symmetry group like $S U(2)_{R}$, however, one needs vector fields that transform in the adjoint representation of the gauge group.

The only way to solve this problem is to identify $S U(2)_{R}$ with an $S U(2)$ subgroup of the scalar manifold isometry group, $G$, and to gauge both $S U(2)$ 's simultaneously. In other words, $S U(2)_{R}$ cannot be gauged by itself, rather one has to gauge a diagonal subgroup of $S U(2)_{R} \times S U(2)_{G} \subset S U(2)_{R} \times G$. The most natural starting point for a gauging of $S U(2)_{R}$ is therefore a "Yang-MillsEinstein supergravity theory" (see $[15,14,21]$ for details on this terminology) in which a subgroup $K \supset S U(2)_{G}$ of $G$ is gauged. In order to be as general as possible, we consider the case when the supersymmetric gauging of $K \subset G$ requires the introduction of tensor fields (the case without tensor fields can easily be recovered as a special case). At this point we require the gauge group $K$ only to have an $S U(2)$ subgroup $S U(2)_{G} \subset K$, but leave it otherwise undetermined.

We start by recalling some relevant properties of YangMills-Einstein supergravity theories with tensor fields (see $[14,21]$ for details). Yang-Mills-Einstein supergravity theories with tensor fields describe the coupling of $n$ vector multiplets and $m$ self-dual tensor multiplets to supergravity. Consequently, the field content of these theories is

$$
\left\{e_{\mu}^{m}, \Psi_{\mu}^{i}, A_{\mu}^{I}, B_{\mu \nu}^{M}, \lambda^{i \tilde{a}}, \varphi^{\tilde{x}}\right\}
$$

where

$$
\begin{gathered}
I, J, K \ldots=0,1, \ldots n \\
M, N, P \ldots=1,2, \ldots 2 m
\end{gathered}
$$

$$
\begin{gathered}
\tilde{a}, \tilde{b}, \tilde{c}, \ldots=1, \ldots, \tilde{n} \\
\tilde{x}, \tilde{y}, \tilde{z}, \ldots=1, \ldots, \tilde{n},
\end{gathered}
$$

with $\tilde{n}=n+2 m$

Note that we have combined the "graviphoton" with the $n$ vector fields of the $n$ vector multiplets into a single ( $n$ +1 )-plet of vector fields $A_{\mu}^{I}$ labeled by the index $I$. Also, the spinor and scalar fields of the vector and tensor multiplets are combined into $\tilde{n}$-tupels of spinor and scalar fields. The indices $\tilde{a}, \tilde{b}, \ldots$ and $\tilde{x}, \tilde{y}, \ldots$ are the flat and curved indices, respectively, of the $\tilde{n}$-dimensional target manifold, $\mathcal{M}$, of the scalar fields. The metric, vielbein and spin connection on $\mathcal{M}$ will be denoted by $g_{\tilde{x} \tilde{y}}, f_{\tilde{x}}^{\tilde{a}}$ and $\Omega_{\tilde{x}}^{\tilde{a} \tilde{b}}$, respectively.

A subset of the vector fields $A_{\mu}^{I}$ is used to promote a subgroup $K$ of the isometry group of $\mathcal{M}$ to a Yang-Millstype gauge symmetry. Apart from these gauge fields, only the tensor fields $B_{\mu \nu}^{M}$, the spin-1/2 fields $\lambda^{i \tilde{a}}$ and the scalar fields $\varphi^{\tilde{x}}$ transform non-trivially under this gauge group $K$.

The $K$-gauge covariant derivatives of these fields are as follows ( $\nabla$ denotes the ordinary spacetime covariant derivative, and $g$ is the coupling constant of the gauge group $K)$

$$
\begin{aligned}
\mathcal{D}_{\mu} \lambda^{i \tilde{a}} & \equiv \nabla_{\mu} \lambda^{i \tilde{a}}+g A_{\mu}^{I} L_{I}^{\tilde{a} \tilde{b}} \lambda^{i \tilde{b}} \\
\mathcal{D}_{\mu} \varphi^{\tilde{x}} & \equiv \partial_{\mu} \varphi^{\tilde{x}}+g A_{\mu}^{I} K_{I}^{\tilde{x}} \\
\mathcal{D}_{\mu} B_{\nu \rho}^{M} & \equiv \nabla_{\mu} B_{\nu \rho}^{M}+g A_{\mu}^{I} \Lambda_{I N}^{M} B_{\nu \rho}^{N} .
\end{aligned}
$$

Here, $K_{I}^{\tilde{x}}$ are the Killing vector fields on $\mathcal{M}$ that generate the subgroup $K$ of its isometry group. The $\varphi$-dependent matrices $L_{I}^{\tilde{a} \widetilde{b}}$ and the constant matrices $\Lambda_{I N}^{M}$ are the $K$-transformation matrices of $\lambda^{i \tilde{a}}$ and $B_{\mu \nu}^{M}$, respectively.

Denoting the curls of $A_{\mu}^{I}$ by $F_{\mu \nu}^{I}$ and the structure constants of $K$ by $f_{J K}^{I}$, we combine the non-Abelian field strengths $\mathcal{F}_{\mu \nu}^{I}=F_{\mu \nu}^{I}+g f_{J K}^{I} A_{\mu}^{J} A_{\nu}^{K}$ with the antisymmetric tensor fields $B_{\mu \nu}^{M}$ to form the tensorial quantity

$$
\mathcal{H}_{\mu \nu}^{\tilde{I}}:=\left(\mathcal{F}_{\mu \nu}^{I}, B_{\mu \nu}^{M}\right) \quad(\widetilde{I}, \widetilde{J}, \widetilde{K}, \ldots=0, \ldots, n+2 m) .
$$

The general Lagrangian of a Yang-Mills-Einstein supergravity theory with tensor fields is then given by [14]

$$
\begin{aligned}
e^{-1} \mathcal{L}= & -\frac{1}{2} R(\omega)-\frac{1}{2} \bar{\Psi}_{\mu}^{i} \Gamma^{\mu \nu \rho} \nabla_{\nu} \Psi_{\rho i}-\frac{1}{4} a_{\tilde{I} \tilde{J}} \mathcal{H}_{\mu \nu}^{\tilde{I}} \mathcal{H}^{\tilde{J} \mu \nu}-\frac{1}{2} \bar{\lambda}^{i \tilde{a}}\left(\Gamma^{\mu} \mathcal{D}_{\mu} \delta^{\tilde{a} \tilde{b}}+\Omega_{\tilde{x}}^{\tilde{a} \tilde{b}} \Gamma^{\mu} \mathcal{D}{ }_{\mu} \varphi^{\tilde{x}}\right) \lambda_{i}^{\tilde{b}}-\frac{1}{2} g_{\tilde{x y}}^{\tilde{y}}\left(\mathcal{D}_{\mu} \varphi^{\tilde{x}}\right)\left(\mathcal{D}^{\mu} \varphi^{\tilde{y}}\right) \\
& -\frac{i}{2} \bar{\lambda}^{i \tilde{a}} \Gamma^{\mu} \Gamma^{\nu} \Psi_{\mu i} f_{\tilde{x}}^{\tilde{a}} \mathcal{D}{ }_{\nu} \varphi^{\tilde{x}}+\frac{1}{4} h_{\tilde{I}}^{\tilde{a}} \bar{\lambda}^{i \tilde{a}} \Gamma^{\mu} \Gamma^{\lambda \rho} \Psi_{\mu i} \mathcal{H}_{\lambda \rho}^{\tilde{I}}+\frac{i}{2 \sqrt{6}}\left(\frac{1}{4} \delta_{\tilde{a} \tilde{b}}^{\tilde{L}} h_{\tilde{I}}+T_{\tilde{a} \tilde{b} \tilde{c}} h_{\tilde{I}}^{\tilde{c}}\right) \bar{\lambda}^{i \tilde{a}} \Gamma^{\mu \nu} \lambda_{i}^{\tilde{b}} \mathcal{H}_{\mu \nu}^{\tilde{I}}
\end{aligned}
$$




$$
\begin{aligned}
& -\frac{3 i}{8 \sqrt{6}} h_{\tilde{I}}\left[\bar{\Psi}_{\mu}^{i} \Gamma^{\mu \nu \rho \sigma} \Psi_{\nu i} \mathcal{H}_{\rho \sigma}^{\tilde{I}}+2 \bar{\Psi}^{\mu i} \Psi_{i}^{\nu} \mathcal{H}_{\mu \nu}^{\tilde{I}}\right]+\frac{e^{-1}}{6 \sqrt{6}} C_{I J K} \varepsilon^{\mu \nu \rho \sigma \lambda} \\
& \times\left\{F_{\mu \nu}^{I} F_{\rho \sigma}^{J} A_{\lambda}^{K}+\frac{3}{2} g F_{\mu \nu}^{I} A_{\rho}^{J}\left(f_{L F}^{K} A_{\sigma}^{L} A_{\lambda}^{F}\right)+\frac{3}{5} g^{2}\left(f_{G H}^{J} A_{\nu}^{G} A_{\rho}^{H}\right)\left(f_{L F}^{K} A_{\sigma}^{L} A_{\lambda}^{F}\right) A_{\mu}^{I}\right\} \\
& +\frac{e^{-1}}{4 g} \varepsilon^{\mu \nu \rho \sigma \lambda} \Omega_{M N} B_{\mu \nu}^{M} \mathcal{D}_{\rho} B_{\sigma \lambda}^{N}+g \bar{\lambda}^{-i \tilde{a}} \Gamma^{\mu} \Psi_{\mu i} W_{\tilde{a}}+g \bar{\lambda}^{i \tilde{a}} \lambda_{i}^{\tilde{b}} W_{\tilde{a} \tilde{b}}-g^{2} P
\end{aligned}
$$

with $e \equiv \operatorname{det}\left(e_{\mu}^{m}\right)$. The transformation laws are (to leading order in fermion fields)

$$
\begin{aligned}
\delta e_{\mu}^{m}= & \frac{1}{2} \bar{\varepsilon}^{i} \Gamma^{m} \Psi_{\mu i} \\
\delta \Psi_{\mu}^{i}= & \nabla_{\mu} \varepsilon^{i}+\frac{i}{4 \sqrt{6}} h_{\tilde{I}}\left(\Gamma_{\mu}^{\nu \rho}-4 \delta_{\mu}^{\nu} \Gamma^{\rho}\right) \mathcal{H}_{\nu \rho}^{\tilde{I}} \varepsilon^{i} \\
\delta A_{\mu}^{I}= & \vartheta_{\mu}^{I} \\
\delta B_{\mu \nu}^{M}= & 2 \mathcal{D}_{[\mu} \vartheta_{\nu]}^{M}+\frac{\sqrt{6} g}{4} \Omega^{M N} h_{N} \bar{\Psi}_{[\mu}^{i} \Gamma_{\nu]} \varepsilon_{i} \\
& +\frac{i g}{4} \Omega^{M N} h_{N \tilde{a}} \tilde{\lambda}^{i \tilde{a}} \Gamma_{\mu \nu} \varepsilon_{i} \\
\delta \lambda^{i \tilde{a}}= & -\frac{i}{2} f_{\tilde{x}}^{\tilde{a}} \Gamma^{\mu}\left(\mathcal{D}_{\mu} \varphi^{\tilde{x}}\right) \varepsilon^{i}+\frac{1}{4} h_{\tilde{I}}^{\tilde{a}} \Gamma^{\mu \nu} \varepsilon^{i} \mathcal{H}_{\mu \nu}^{\tilde{I}}+g W^{\tilde{a}} \varepsilon^{i} \\
\delta \varphi^{\tilde{x}}= & \frac{i}{2} f_{\tilde{a}}^{\tilde{x}-\varepsilon^{i} \lambda_{i}^{\tilde{a}}}
\end{aligned}
$$

with

$$
\vartheta_{\mu}^{\tilde{I}} \equiv-\frac{1}{2} h_{\tilde{a}}^{\tilde{I}} \bar{\varepsilon} \bar{\varepsilon}_{\mu} \Gamma_{\mu} \lambda_{i}^{\tilde{a}}+\frac{i \sqrt{6}}{4} h^{\tilde{I}} \bar{\Psi}_{\mu}^{i} \varepsilon_{i} .
$$

The various scalar field dependent quantities $\stackrel{\circ}{a_{\tilde{I}}}, h_{\tilde{I}}, h^{\tilde{I}}$, $h_{\tilde{I}}^{\tilde{a}}, h^{\tilde{I} \tilde{a}}$ and $T_{\tilde{a} \tilde{b} \tilde{c}}$ that contract the different types of indices are already present in the corresponding ungauged MESGT's and describe the "very special" geometry of the scalar manifold $\mathcal{M}$ (see [13] for details). These ungauged MESGT's also contain a constant symmetric tensor $C_{\tilde{I} \widetilde{J} \tilde{K}}$. If the gauging of $K$ involves the introduction of tensor fields, the coefficients of the type $C_{M N P}$ and $C_{I J M}$ have to vanish [14]. The only components that survive such a gauging are thus $C_{I J K}$, which appear in the Chern-Simons-like term of Eq. (2.3), and $C_{I M N}$, which are related to the transformation matrices of the tensor fields by

$$
\Lambda_{I N}^{M}=\frac{2}{\sqrt{6}} \Omega^{M P} C_{I P N} .
$$

Here $\Omega^{M N}$ is the inverse of $\Omega_{M N}$, which is a (constant) invariant antisymmetric tensor of the gauge group $K$ :

$$
\Omega_{M N}=-\Omega_{N M}, \quad \Omega_{M N} \Omega^{N P}=\delta_{M}^{P} .
$$

The quantities $W^{\tilde{a}}(\varphi)$ and $W^{\tilde{a} \tilde{b}}(\varphi)$ and the scalar potential $P(\varphi)$ are due to the gauging of $K$ in the presence of the tensor fields, and are given by

$$
\begin{aligned}
W^{\tilde{a}} & =-\frac{\sqrt{6}}{8} h_{M}^{\tilde{a}} \Omega^{M N} h_{N} \\
W^{\tilde{a} \tilde{b}} & =-W^{\tilde{b} \tilde{a}}=i h^{J[\tilde{a}} K_{J}^{\tilde{b}]}+\frac{i \sqrt{6}}{4} h^{J} K_{J}^{\tilde{a} ; \tilde{b}} \\
P & =2 W^{\tilde{a}} W^{\tilde{a}},
\end{aligned}
$$

where the semicolon denotes covariant differentiation on the target space $\mathcal{M}$.

We will now use the above theory as our starting point for the additional gauging of $S U(2)_{R}$. To this end, we first split the index $I$ of the $(n+1)$ vector fields $A_{\mu}^{I}$ according to

$$
I=\left(A, I^{\prime}\right)
$$

where $A, B, C, \ldots \in\{1,2,3\}$ are the indices corresponding to the three gauge fields of $S U(2)_{G} \subset K$, and $I^{\prime}, J^{\prime}, K^{\prime}, \ldots$ label the remaining $(n-2)$ vector fields.

In order to gauge $S U(2)_{R}$, we use the gauge fields $A_{\mu}^{A}$ to covariantize the $K$ - and spacetime covariant derivatives of the fermions also with respect to $S U(2)_{R}$; i.e., we make the replacements

$$
\begin{aligned}
\nabla_{\mu} \Psi_{\nu}^{i} \rightarrow \mathfrak{D}_{\mu} \Psi_{\nu}^{i} & :=\nabla_{\mu} \Psi_{\nu}^{i}+g_{R} A_{\mu}^{A} \Sigma_{A j}^{i} \Psi_{\nu}^{j} \\
\nabla_{\mu} \varepsilon^{i} \rightarrow \mathfrak{D}_{\mu} \varepsilon^{i} & :=\nabla_{\mu} \varepsilon^{i}+g_{R} A_{\mu}^{A} \Sigma_{A j}^{i} \varepsilon^{j} \\
\mathcal{D}_{\mu} \lambda^{i \tilde{a}} \rightarrow \mathfrak{D}_{\mu} \lambda^{i \tilde{a}} & :=\mathcal{D}_{\mu} \lambda^{i \tilde{a}}+g_{R} A_{\mu}^{A} \Sigma_{A j}^{i} \lambda^{j \tilde{a}} \\
& \equiv \nabla_{\mu} \lambda^{i \tilde{a}}+g A_{\mu}^{I} L_{I}^{\tilde{a} \tilde{b}} \lambda^{i \tilde{b}}+g_{R} A_{\mu}^{A} \Sigma_{A j}^{i} \lambda^{j \tilde{a}}
\end{aligned}
$$

in the Lagrangian (2.3) and the transformation laws (2.4). Here, $g_{R}$ denotes the $S U(2)_{R}$ coupling constant, and the $\Sigma_{A j}^{i}$ $(i, j, \ldots=1,2)$ are the $S U(2)_{R}$ transformation matrices of the fermions, which can be chosen as 


$$
\Sigma_{A j}^{i}=\frac{i}{2} \sigma_{A j}^{i}
$$

with $\sigma_{A j}^{i}$ being the Pauli matrices. The indices $i, j, \ldots$ are raised and lowered according to

$$
X^{i}=\varepsilon^{i j} X_{j}, \quad X_{i}=X^{j} \varepsilon_{j i}
$$

with $\varepsilon^{i j}, \varepsilon_{i j}$ antisymmetric and $\varepsilon^{12}=\varepsilon_{12}=1$. (The tracelessness of $\Sigma_{A j}^{i}$ then implies $\Sigma_{A i j}=\Sigma_{A j i}$.)

The above replacements break supersymmetry, but the latter can be restored by adding

$$
\begin{aligned}
e^{-1} \mathcal{L}^{\prime}= & g_{R} \bar{\Psi}_{\mu}^{i} \Gamma^{\mu \nu} \Psi_{\nu}^{j} R_{0 i j}(\varphi)+g_{R} \bar{\lambda}^{i \tilde{a}} \Gamma^{\mu} \Psi_{\mu}^{j} R_{\tilde{a} i j}(\varphi) \\
& +g_{R} \bar{\lambda}^{-i \tilde{a}} \lambda^{j \tilde{b}} R_{\tilde{a} \tilde{b} i j}(\varphi)-g_{R}^{2} P^{(R)}(\varphi)
\end{aligned}
$$

to the Lagrangian and by adding

$$
\begin{aligned}
\delta^{\prime} \Psi_{\mu i} & =\frac{2}{3} g_{R} R_{0 i j}(\varphi) \Gamma_{\mu} \varepsilon^{j} \\
\delta^{\prime} \lambda_{i}^{\tilde{a}} & =g_{R} R_{i j}^{\tilde{a}}(\varphi) \varepsilon^{j}
\end{aligned}
$$

to the transformation laws.

The quantities $R_{0 i j}, R_{i j}^{\tilde{a}}, R_{\tilde{a} \tilde{b} i j}$ and the additional potential term $P^{(R)}$ are fixed by supersymmetry:

$$
\begin{aligned}
R_{0 i j} & =i \sqrt{\frac{3}{8}} h^{A} \Sigma_{A i j} \\
R_{i j}^{\tilde{a}} & =h^{A \tilde{a}} \Sigma_{A i j} \\
R_{\tilde{a} \tilde{b} i j} & =-\frac{1}{3} \delta_{\tilde{a} \tilde{b}} R_{0 i j}-i \sqrt{\frac{2}{3}} T_{\tilde{a} \tilde{b} \tilde{c}} R_{i j}^{\tilde{c}} \\
P^{(R)} & =-\frac{16}{3} R_{0 j}^{i} R_{0 i}^{j}-R_{j}^{\tilde{a} i} R_{i}^{\tilde{a} j} .
\end{aligned}
$$

Supersymmetry also requires

$$
\begin{aligned}
f_{I^{\prime} B}^{A}=f_{I^{\prime} J^{\prime}}^{A} & =0 \\
g_{R}\left[\Sigma_{A}, \Sigma_{B}\right]_{i j} & =g f_{A B}^{C} \Sigma_{C i j} \\
\Sigma_{A i j, \tilde{x}} & =0 .
\end{aligned}
$$

[The structure constants of the type $f_{I^{\prime} A}^{J^{\prime} A}$ do not necessarily have to vanish for supersymmetry. If they do vanish, however, $K$ is a direct product of $S U(2)_{G}$ and some other group $K^{\prime}$. Otherwise, $K$ is a semi-direct product of the form $\left(S U(2)_{G} \times S\right) \ltimes T$, where $\ltimes$ denotes the semi-direct product and $S$ and $T$ are some other subgroups of $K$.]

The following constraints are consequences of the above and are needed in the proof of supersymmetry:

$$
R_{i j}^{\tilde{a}} K_{J}^{\tilde{a}}=-\sqrt{\frac{3}{2}} \Sigma_{A i j} f_{J K}^{A} h^{K}
$$

$$
-i \sqrt{\frac{3}{2}} h^{B \tilde{a}} f_{B C}^{A} h^{C} \Sigma_{A i j}=\frac{10}{3} W^{\tilde{a}} R_{0 i j}+2 R_{i j}^{\tilde{b}} W_{\tilde{a} \tilde{b}}+2 R_{\tilde{a} \tilde{b} i j} W^{\tilde{b}}
$$

$$
\begin{aligned}
R_{i j ; \tilde{x}}^{\tilde{a}} & =i f_{\tilde{x}}^{\tilde{a}} R_{0 i j}-i R_{\tilde{a} \tilde{b} i j} f_{\tilde{x}}^{\tilde{b}} \\
R_{0 i j, \tilde{x}} & =-\frac{i}{2} R_{\tilde{x} i j} .
\end{aligned}
$$

Furthermore, the cancellation of the $\delta \varphi^{\tilde{x}}$ variation of $P^{(R)}$ and similar terms requires that

$$
\operatorname{tr}\left(\Sigma_{(A} \Sigma_{B)} \Sigma_{C}\right)=0
$$

[as well as $\operatorname{tr}\left(\Sigma_{A}\right)=0$ ], which is, however, a general property of traceless anti-Hermitian $(2 \times 2)$ matrices.

For the sake of concreteness, let us conclude this section with a brief overview of the most interesting examples of Yang-Mills-Einstein supergravity theories that admit the gauging of $S U(2)_{R}$.

Even though the constraints from supersymmetry [Eq. (2.18)] allow $K$ to be a semi-direct product group, we shall restrict ourselves to gauge groups that are not of the semidirect type. In this case, $K$ is a direct product of $S U(2)_{G}$ with another group. We can thus confine ourselves to the case $K=S U(2)_{G}$, since additional group factors in $K$ do not change the structure of the above theory very much.

Now, to be able to gauge $K=S U(2)_{G}$, the isometry group of $\mathcal{M}$ must have an $S U(2)$ subgroup, $S U(2)_{G}$, that extends to a symmetry group of the full Lagrangian with three of the vector fields of the theory transforming in the adjoint representation of $S U(2)_{G}$.

For the generic Jordan family of MESGT's with the scalar manifold $S O(1,1) \times S O(\tilde{n}-1,1) / S O(\tilde{n}-1)$ [13], such a subgroup exists for all theories with $\tilde{n}>3$ (see Sec. III A). Similarly, for the generic non-Jordan family with the scalar manifold $S O(\tilde{n}, 1) / S O(\tilde{n})$ [22] one can gauge $S U(2)_{G}$ whenever $\tilde{n}>3$ (see Sec. III C).

Of the "magical" $\mathcal{N}=2$ MESGT's [13], all but the one defined by the Jordan algebra of real symmetric $(3 \times 3)$ matrices, $J_{3}^{\mathrm{R}}$, admit such a gauging.

Finally, all the members of the infinite family with $S U(N)$ isometries $(N>3)$ described in Ref. [14] also admit a gauging of $S U(2)_{G}$ [and thus of $S U(2)_{R}$ ].

For the generic Jordan and the generic non-Jordan families one can choose the $S U(2)_{G}$ subgroup of the isometry group such that all the other vector fields are inert under it, i.e. one does not have to dualize any vector fields to tensor fields. On the other hand, the gauging of $S U(2)_{G}$ requires the dualization of some of the vector fields to tensor fields in the magical theories as well as in the theories with $S U(N)$ isometries.

\section{THE SCALAR POTENTIAL}

As seen in the previous section, the gauging of $S U(2)_{R}$ introduces an additional contribution, $P^{(R)}(\varphi)$, to the total scalar potential. Before we take a closer look at this poten- 
tial, let us first make contact with the earlier work [15] on the most general gauging of a $U(1)_{R}$ subgroup of $S U(2)_{R}$. We first note that the triplet of vector fields transforming in the adjoint representation of $S U(2)_{G}$ cannot include the graviphoton. This follows from the fact that $S U(2)_{G}$ is a subgroup of the compact part of the isometry group of $\mathcal{M}$, under which the graviphoton is inert. Thus the $U(1)_{R}$ gauged theories obtained by restricting oneself to a $U(1)$ subgroup of $S U(2)_{R}$ do not describe the most general $U(1)_{R}$ gaugings possible: For the most general $U(1)_{R}$ gauging, one can choose an arbitrary linear combination $A_{\mu}^{I} V_{I}$ of all the vector fields, as was done in [15], including the graviphoton.

For the theories of the Jordan family, it was shown in [15] that the generic $U(1)_{R}$ gauging either leads to a flat potential with Minkowski ground states whenever $V_{I}$ corresponds to an idempotent of the Jordan algebra, or an anti-de Sitter ground state whenever $V_{I}$ lies in the "domain of positivity" of the Jordan algebra, or to no critical points at all when none of the above is true for $V_{I}$. Looking now at the $U(1)_{R}$ restrictions of the $S U(2)_{R}$ gaugings in the Jordan family, one finds that the $V_{I}$ are of the last type, i.e., they are neither idempotents nor do they lie in the domain of positivity. This already suggests that, at least in the Jordan family, the $S U(2)_{R}$ gauging leads to theories without critical points.

In fact, we are able to verify this statement for all theories for which the scalar manifold $\mathcal{M}$ is a symmetric space. These can be divided into three families:

(i) The generic Jordan family.

(ii) The magical Jordan family.

(iii) The generic non-Jordan family.

Before we look at each of these three families in more detail, let us recast the scalar potential $P^{(R)}$ of Eq. (2.17) into a more compact form. In the basis $(2.11), P^{(R)}$ becomes

$$
P^{(R)}=\left[-h^{A} h^{B}+\frac{1}{2} h^{A \tilde{a}} h^{B \tilde{a}}\right] \delta_{A B} .
$$

Using [13]

$$
C_{\tilde{I} J \tilde{K}} h^{\tilde{K}}=h_{\tilde{I}} h_{\tilde{J}}-\frac{1}{2} h_{\tilde{I}}^{\tilde{a}} h_{\tilde{J}}^{\tilde{a}},
$$

this can be rewritten as

$$
P^{(R)}=-C^{A B \widetilde{I}} h_{\tilde{I}} \delta_{A B},
$$

where we have defined

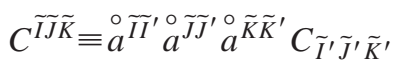

with $\stackrel{\circ}{a} \widetilde{I J}^{J}$ being the inverse of $\stackrel{\circ}{a} \widetilde{I J}$.

Let us now analyze this potential for the above-mentioned three families of symmetric spaces.

\section{A. The generic Jordan family}

The generic Jordan family corresponds to the scalar manifolds of the form $\mathcal{M}=S O(1,1) \times S O(\tilde{n}-1,1) / S O(\tilde{n}-1)$. The latter can be described as the hypersurface $N(\xi)=1$ of the cubic polynomial [13]

$$
\begin{aligned}
N(\xi) & =\left(\frac{2}{3}\right)^{3 / 2} C_{\tilde{I} \tilde{J} \tilde{K}} \xi^{\tilde{I}} \xi^{\tilde{J}} \xi^{\tilde{K}} \\
& =\sqrt{2} \xi^{0}\left[\left(\xi^{1}\right)^{2}-\left(\xi^{2}\right)^{2}-\cdots-\left(\xi^{\tilde{n}}\right)^{2}\right],
\end{aligned}
$$

where the $\xi^{\tilde{I}}$ parametrize an ambient space $\mathbb{R}^{\tilde{n}+1}$. The isometry group of this space is $S O(1,1) \times S O(\tilde{n}-1,1)$. For $S U(2) \sim S O(3)$ to be a subgroup, one obviously needs $\tilde{n}$ $\geqslant 4$, as we will assume from now on.

The constraint $N(\xi)=1$ can be solved by

$$
\begin{gathered}
\xi^{0}=\frac{1}{\sqrt{2}\|\varphi\|^{2}} \\
\xi^{1}=\varphi^{1} \\
\vdots \\
\xi^{\tilde{n}}=\varphi^{\tilde{n}},
\end{gathered}
$$

where $\|\varphi\|^{2} \equiv\left(\varphi^{1}\right)^{2}-\left(\varphi^{2}\right)^{2}-\ldots-\left(\varphi^{\tilde{n}}\right)^{2}$ has been introduced. As explained in [21], the scalar field metric $g_{x \tilde{x y}}$ and the vector field metric $\stackrel{\circ}{a_{\tilde{I} \tilde{J}}}$ are positive definite only for $\|\varphi\|^{2}>0$. Without loss of generality, we choose $A_{\mu}^{2}, A_{\mu}^{\mathcal{3}}, A_{\mu}^{4}$ as the $S O(3)$ gauge fields.

For the Jordan cases, one has $C_{\tilde{I} \widetilde{K} \widetilde{K}}=C^{\tilde{I} \widetilde{K}}=$ const $(\mathrm{com}-$ ponentwise) [13]. Using $h_{\tilde{I}}=\left.(1 / \sqrt{6})\left(\partial / \partial \xi^{\tilde{I}}\right) N\right|_{N=1}$ [13], one then obtains for the scalar potential (3.2)

$$
P^{(R)}=\frac{3}{2}\|\varphi\|^{2}
$$

It is easy to see that this scalar potential does not have any critical points in the physically relevant region $\|\varphi\|^{2}>0$.

This situation does not change when one gauges an additional $S O(2) \subset G$ along the lines of Ref. [21] by introducing tensor fields. For such a gauging, one needs at least $\tilde{n} \geqslant 6$. Choosing $\xi^{5}$ and $\xi^{6}$ to form an $S O(2)$ doublet, the corresponding vector fields $A_{\mu}^{5}$ and $A_{\mu}^{6}$ have to be dualized to tensor fields. This gives rise to the additional potential term [21]

$$
P=\frac{1}{8} \frac{\left[\left(\varphi^{5}\right)^{2}+\left(\varphi^{6}\right)^{2}\right]}{\|\varphi\|^{6}} .
$$

It is easy to verify that the combined potential $P_{t o t}=P^{(R)}$ $+P$ does not have any ground states either. 


\section{B. The magical Jordan family}

We now turn to the magical Jordan family [13]. The simplest example in which $S U(2)_{R}$ can be gauged is provided by the model with the scalar manifold $\mathcal{M}$ $=S L(3, \mathrm{C}) / S U(3)$. This theory contains eight vector multiplets (i.e. it comprises eight scalar fields and nine vector fields). $\mathcal{M}$ can be described as the hypersurface $N(\xi)=1$ of the cubic polynomial

$$
N(\xi)=\sqrt{2} \xi^{4} \eta_{\alpha \beta} \xi^{\alpha} \xi^{\beta}+\gamma_{\alpha M N} \xi^{\alpha} \xi^{M} \xi^{N}
$$

where

$$
\begin{aligned}
\alpha, \beta, \ldots & =0,1,2,3 \\
M, N, \ldots= & 5,6,7,8 \\
\eta_{\alpha \beta} & =\operatorname{diag}(+,-,-,-) \\
\gamma_{0} & =-\mathbf{1}_{4} \\
\gamma_{1} & =\mathbf{1}_{2} \otimes \sigma_{1} \\
\gamma_{2} & =-\sigma_{2} \otimes \sigma_{2} \\
\gamma_{3} & =\mathbf{1}_{2} \otimes \sigma_{3} .
\end{aligned}
$$

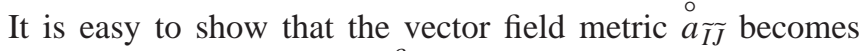
degenerate, when $\eta_{\alpha \beta} \xi^{\alpha} \xi^{\beta}=0$. We therefore can restrict ourselves to the region $\eta_{\alpha \beta} \xi^{\alpha} \xi^{\beta} \neq 0$, where the constraint $N(\xi)=1$ can be solved by

$$
\begin{gathered}
\xi^{\alpha}=\varphi^{\alpha}=: x^{\alpha} \\
\xi^{4}=\frac{1-b^{T} \bar{x} b}{\sqrt{2}\|x\|^{2}} \\
\xi^{M}=\varphi^{M}=: b^{M},
\end{gathered}
$$

where $b^{T} \bar{x} b \equiv b^{M} \bar{x}_{M N} b^{N}$ with $\quad \bar{x}_{M N} \equiv x^{\alpha} \gamma_{\alpha M N} \quad$ and $\|x\|^{2}$ $\equiv \eta_{\alpha \beta} x^{\alpha} x^{\beta}$.

In the above model, one can gauge a $[U(1) \times S U(2)]$ subgroup of the isometry group $S L(3, \mathrm{C})$. The vector field $A_{\mu}^{0}$ corresponds to the $U(1)$ gauge field, whereas the vector fields $A_{\mu}^{1}, A_{\mu}^{2}, A_{\mu}^{3}$ act as the $S U(2)$ gauge fields. The vector fields $A_{\mu}^{M}$ are charged under $[U(1) \times S U(2)]$ and have to be dualized to tensor fields. The vector field $A_{\mu}^{4}$ is a spectator vector field. The introduction of the tensor fields leads to a non-trivial potential $P$, which turns out to be

$$
P=-\frac{1}{8} b^{T}(\bar{x})^{3} b
$$

As described earlier, the $S U(2)_{G}$ gauge fields $A_{\mu}^{1}, A_{\mu}^{2}, A_{\mu}^{3}$ can be used to simultaneously gauge $S U(2)_{R}$. This leads to an additional potential

$$
P^{(R)}=\frac{3}{2}\|x\|^{2}
$$

Taking into account that $\operatorname{det}\left((\bar{x})^{3}\right)=\left[\|x\|^{2}\right]^{6}$, it easy to verify that the total potential $P_{\text {tot }}=P+P^{(R)}$ does not have any critical points in the physically relevant region, where $\|x\|^{2} \neq 0$.

The other magical theories corresponding to $\mathcal{M}$ $=S U^{*}(6) / U S p(6)$ and $\mathcal{M}=E_{6(-26)} / F_{4}$, which also allow the gauging of $S U(2)_{R}$, have a very similar structure to the above and contain the $S L(3, \mathrm{C}) / S U(3)$ model as a subsector; one therefore does not expect to find any critical points either.

\section{The generic non-Jordan family}

This leaves us with the theories of the generic non-Jordan family [22]. They are given by $\mathcal{M}=S O(1, \tilde{n}) / S O(\tilde{n})$, which can be described as the hypersurface $N(\xi)=1$ of

$$
N(\xi)=\sqrt{2} \xi^{0}\left(\xi^{1}\right)^{2}-\xi^{1}\left[\left(\xi^{2}\right)^{2}+\cdots+\left(\xi^{\tilde{n}}\right)^{2}\right] .
$$

The constraint $N=1$ can be solved by

$$
\begin{aligned}
\xi^{0} & =\frac{1}{\sqrt{2}\left(\varphi^{1}\right)^{2}}+\frac{1}{\sqrt{2}} \varphi^{1}\left[\left(\varphi^{2}\right)^{2}+\ldots+\left(\varphi^{\tilde{n}}\right)^{2}\right] \\
\xi^{1} & =\varphi^{1} \\
\xi^{2} & =\varphi^{1} \varphi^{2} \\
& \vdots \\
\xi^{\tilde{n}} & =\varphi^{1} \varphi^{\tilde{n}} .
\end{aligned}
$$

In contrast to the Jordan families, one no longer has the equality of the constant $C_{\widetilde{I} \widetilde{J} \widetilde{K}}$ to $C^{\widetilde{I J} \widetilde{K}}$. Instead, the $C^{\widetilde{I J} \widetilde{K}}$ are now scalar field dependent, which makes a similar analysis more complicated. What makes the calculation of the scalar potential nevertheless feasible is that the scalar field metric $g_{x \tilde{y}}$ becomes diagonal, and therefore easily invertible, in the above coordinate system. To be specific, one obtains

$$
g_{\tilde{x y}}=\operatorname{diag}\left[3 /\left(\varphi^{1}\right)^{2},\left(\varphi^{1}\right)^{3}, \ldots,\left(\varphi^{1}\right)^{3}\right],
$$

which is positive definite for $\varphi^{1}>0$

In order to gauge an $S O(3) \sim S U(2)$ subgroup of the isometry group of $\mathcal{M}$, one obviously needs at least $\tilde{n} \geqslant 4$, as we will assume from now on. We choose $A_{\mu}^{2}, A_{\mu}^{3}, A_{\mu}^{4}$ as the $S U(2)_{G}$ gauge fields. Inspection of $N$ above shows that this group rotates $\xi^{2}, \xi^{3}, \xi^{4}$ into each other, but leaves the other $\xi^{\tilde{I}}$ unchanged. Thus, no tensor fields have to be introduced. The resulting scalar potential (3.1) turns out to be

$$
P^{(R)}=-\frac{1}{2}\left(\varphi^{1}\right)^{2}\left[\left(\varphi^{2}\right)^{2}+\left(\varphi^{3}\right)^{2}+\left(\varphi^{4}\right)^{2}\right]+\frac{3}{2} \frac{1}{\varphi^{1}},
$$

which again does not admit any ground states for the physically interesting region $\varphi^{1}>0$.

Similar conclusions hold true when one introduces tensor fields by gauging an additional $S O(2)$, which is possible 
when $\tilde{n}>5$. Just as in the generic Jordan case, this $S O(2)$ can be chosen to rotate $\xi^{5}$ and $\xi^{6}$ into each other and thus requires the dualization of $A_{\mu}^{5}$ and $A_{\mu}^{6}$ to tensor fields. The total scalar potential then gets an additional contribution, $P$, which turns out to be

$$
P=\frac{1}{8}\left(\varphi^{1}\right)^{5}\left[\left(\varphi^{5}\right)^{2}+\left(\varphi^{6}\right)^{2}\right]
$$

Again, it is easy to see that the combined potential $P_{t o t}=P$ $+P^{(R)}$ does not have any critical points for $\varphi^{1}>0$.

To conclude, at least when the scalar manifold $\mathcal{M}$ is a symmetric space, the $S U(2)_{R}$ gauging leads to a total scalar potential which does not have any critical points.

One also notes that the gauge coupling $g_{R}$ for $S U(2)_{R}$ is related to $g$ (2.19), which is, of course, a consequence of the fact that we are gauging a diagonal subgroup of $S U(2)_{R} \times S U(2)_{G}$. This implies that one cannot tune the relative coupling constants as in the gaugings of $U(1)_{R} \times K$ in order to change the properties of critical points of the scalar potential $P_{t o t}=P+P^{(R)}[14,21]$ (if such critical points were to exist for some of the models we have not studied in this paper). Hence, $S U(2)_{R^{-}}$gauged supergravity theories are much more rigid than their $U(1)_{R}$-gauged relatives.

Note added. This paper appeared contemporaneously with Ref. [23] on the general $\mathcal{N}=2 d=5$ supergravity including hypermultiplets and $S U(2)_{R}$ gauging. Where these two papers overlap, the authors of [23] have found the results of their revised version to be consistent with our results.

\section{ACKNOWLEDGMENTS}

This work was supported in part by the National Science Foundation under Grant Number PHY-9802510. We would like to thank Eric Bergshoeff, Renata Kallosh, Andrei Linde, and Toine van Proeyen for fruitful discussions.
[1] J. Maldacena, Adv. Theor. Math. Phys. 2, 231 (1998).

[2] S. Gubser, I. R. Klebanov, and A. M. Polyakov, Phys. Lett. B 428, 105 (1998).

[3] E. Witten, Adv. Theor. Math. Phys. 2, 253 (1998); 2, 505 (1998).

[4] For an extensive list of references on AdS-CFT dualities, see the recent review paper by O. Aharony, S. S. Gubser, J. Maldacena, H. Ooguri, and Y. Oz, Phys. Rep. 323, 183 (2000).

[5] L. Randall and R. Sundrum, Phys. Rev. Lett. 83, 3370 (1999).

[6] L. Randall and R. Sundrum, Phys. Rev. Lett. 83, 4690 (1999).

[7] R. Altendorfer, J. Bagger, and D. Nemeschansky, Phys. Rev. D (to be published), hep-th/0003117.

[8] T. Gherghetta and A. Pomarol, Nucl. Phys. B586, 141 (2000).

[9] A. Falkowsky, Z. Lalak, and S. Pokorski, Phys. Lett. B 491, 172 (2000).

[10] R. Kallosh and A. Linde, J. High Energy Phys. 02, 005 (2000).

[11] K. Behrndt and M. Cvetič, Phys. Rev. D 61, 101901 (2000).

[12] G. W. Gibbons and N. D. Lambert, Phys. Lett. B 488, 90 (2000).

[13] M. Günaydin, G. Sierra, and P. K. Townsend, Nucl. Phys.
B242, 244 (1984); Phys. Lett. 133B, 72 (1983).

[14] M. Günaydin and M. Zagermann, Nucl. Phys. B572, 131 (2000).

[15] M. Günaydin, G. Sierra, and P. K. Townsend, Nucl. Phys. B253, 573 (1985).

[16] K. Pilch, P. K. Townsend, and P. van Nieuwenhuizen, Phys. Lett. 136B, 38 (1984).

[17] M. Pernici, K. Pilch, and P. van Nieuwenhuizen, Phys. Lett. 143B, 103 (1984).

[18] M. Günaydin, L. J. Romans, and N. P. Warner, Phys. Lett. 154B, 268 (1985)

[19] M. Günaydin, L. J. Romans, and N. P. Warner, Nucl. Phys. B272, 598 (1986).

[20] M. Pernici, K. Pilch, and P. van Nieuwenhuizen, Nucl. Phys. B259, 460 (1985).

[21] M. Günaydin and M. Zagermann, Phys. Rev. D 62, 044028 (2000).

[22] M. Günaydin, G. Sierra, and P. K. Townsend, Class. Quantum Grav. 3, 763 (1986).

[23] A. Ceresole and G. Dall'Agata, Nucl. Phys. B585, 143 (2000). 